\title{
Central Odontogenic Fibroma, its Treatment and its Recurrence: A Systematic Review
}

Review Article

Lombardi-Azócar $\mathrm{JJ}^{1 *}$, García-Cano E ${ }^{1}$, Malagón-Hidalgo $\mathrm{HO}^{2}$, González-Magaña F³ , Montiel-Jarquín AJ ${ }^{4}$, González-Chapa DR ${ }^{1}$

${ }^{1}$ Plastic and Reconstructive Surgery Residtent, ISSEMYM, Toluca, State of México, México.

${ }^{2}$ Head of Plastic and Reconsructive Surgery, ISSEMYM, Toluca, State of México, México.

${ }^{3}$ Maxillofacial Surgery Attending Physician, ISSEMYM, Toluca, State of México, México.

${ }^{4}$ Head of investigation in HTYO IMSS, Puebla, Puebla, México.

\section{Abstract}

Introduction: The central odontogenic fibroma (COF) is an extremely rare benign neoplasm of intraosseous or central presentation, being itself only $0.1 \%$ of all odontogenic tumors. The purpose of this systematic review is to provide an overview of the treatment of the central odontogenic fibroma and its recurrence.

Methods: Following PRISMA guideline, a systematic review was carried out.

Results: The initial search retrieved 51763 articles identified through database searching, and 41 articles identified through manual search. After examination of the title and the abstract, 83 articles were retrieved for further examination. Sixty-two articles were included, thus obtaining a total of 104 patients. The recurrence of the 84 patients was $4.76 \%$.

Discussion: The recurrence reports vary in the literature. The most recent review by Garcia-Cano E, et al., showed no recurrence whatsoever; Ramer and colleagues reported only 5 cases of recurrence. However, only 39 cases out of 68 were followed up. In this systematic review out of 84 patients, 4 (4.7\%) presented recurrence. From these, 3 cases were treated by enucleation and one by curettage.

Conclusion: The recurrence rate in these tumors is very low and mainly related to an incomplete resection. Therefore, conservative surgery must be done as a first-line treatment.

Keywords: Systematic Review; Central Odontogenic Fibroma; Treatment; Recurrence.

\section{Introduction}

The central odontogenic fibroma (COF) is an extremely rare benign neoplasm of intraosseous or central presentation, being itself only $0.1 \%$ of all odontogenic tumors $[1,2]$. It is histologically considered among the odontogenic tumors of mesenchymal tissue, with or without formation of odontogenic epithelium [1]. The World Health Organization (WHO) defines it as a benign proliferation of ectomesenchyme fibroblastic tissue characterized by a variable number of apparently inactive odontogenic epithelium [3]. There are two distinct histological types:

1. Simple COF, characterized as a poorly cellular and myxoid fibroblastic neoplasia without odontogenic epithelial component. 2. WHO or complex COF, with abundant odontogenic epithelium cell with foci of calcification [4].

The treatment of $\mathrm{COF}$ in the literature mainly involves conservative surgery [5]. However, this treatment is based only in case reports or literature reviews without a complete follow-up of all the patients.

The purpose of this systematic review is to provide an overview of the treatment of the central odontogenic fibroma and its recurrence, based on the following key questions:

(1) What are the surgical treatments reported?

(2) What is the recurrence with these treatments?

\section{Methods}

\section{Search Strategy}

A research through public domain databases was performed to identify articles focusing on COF and its treatment by two

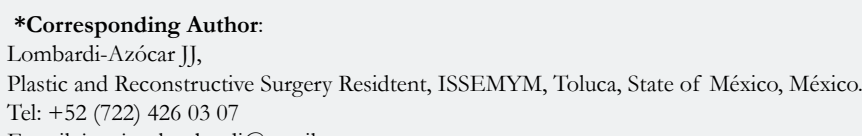

Citation: Lombardi-Azócar JJ, García-Cano E, Malagón-Hidalgo HO, González-Magaña F, Montiel-Jarquín AJ, et al., (2017) Central Odontogenic Fibroma, its Treatment and its Recurrence: A Systematic Review. Int J Dentistry Oral Sci. S10:001, 1-7. doi: http://dx.doi.org/10.19070/2377-8075-SI010001

Copyright: Lombardi-Azócar $\mathbf{J J}^{\circ}$ 2017. This is an open-access article distributed under the terms of the Creative Commons Attribution License, which permits unrestricted use, distribution and reproduction in any medium, provided the original author and source are credited. 
authors Lombardi-Azócar JJand García-Cano E, including the following databases: PubMed, Embase, Cochrane Library, and Web of Science (all searched up to January $4^{\text {th }}, 2016$ ). In addition, we performed a manual search of secondary sources including references of the articles initially identified. The goal was to identify all studies addressing COF in relation to its treatment and its recurrence.

The following search terms were used: $((((($ Central Odontogenic Fibroma) OR COF) AND Treatment) OR Enucleation) OR curettage) OR Resection) AND Recurrence.

\section{Data Extraction and Analysis}

We followed the PRISMA guideline for systematic reviews.

All the articles regarding the prevalence and treatment of patients with COF and its recurrence were included. The full texts of articles that met the inclusion criteria and of articles whose abstract was lacking information were obtained.

Data on the number of patients, patient characteristics such as gender, age, COF localization, correlation with unerupted tooth, treatment used, follow-up, and recurrence were tabulated.

\section{Inclusion Criteria}

Patients with histopathological diagnosis of COF, with follow-up and information regarding recurrence.

\section{Exclusion Criteria}

Patients with histopathological diagnosis of odontogenic fibroma, without diagnosis of being central.

Patients without follow-up.

Patients without information about recurrence. Patients with histopathological findings of $\mathrm{COF}$ and other types in the same tumor. Articles that did not have an English abstract with the above-mentioned characteristics.

\section{Results}

The initial search retrieved 51763 articles identified through database searching, and 41 articles identified through manual search. After examination of the title and the abstract, 83 articles were retrieved for further examination. Sixty-two articles were included, thus obtaining a total of 104 patients (Table 1).

For the analysis of the correlation with an unerupted tooth, 13 patients from one article were excluded because no information was available, resulting in a total of 91 patients.

For the analysis of the tumor localization, all of the 104 patients were included.

For the treatment and recurrence analysis, 20 patients from 5 articles were excluded due to a lack of information about followup, diagnosis of being central, or information about recurrence.

Figure 1. Data Search Flow Chart.

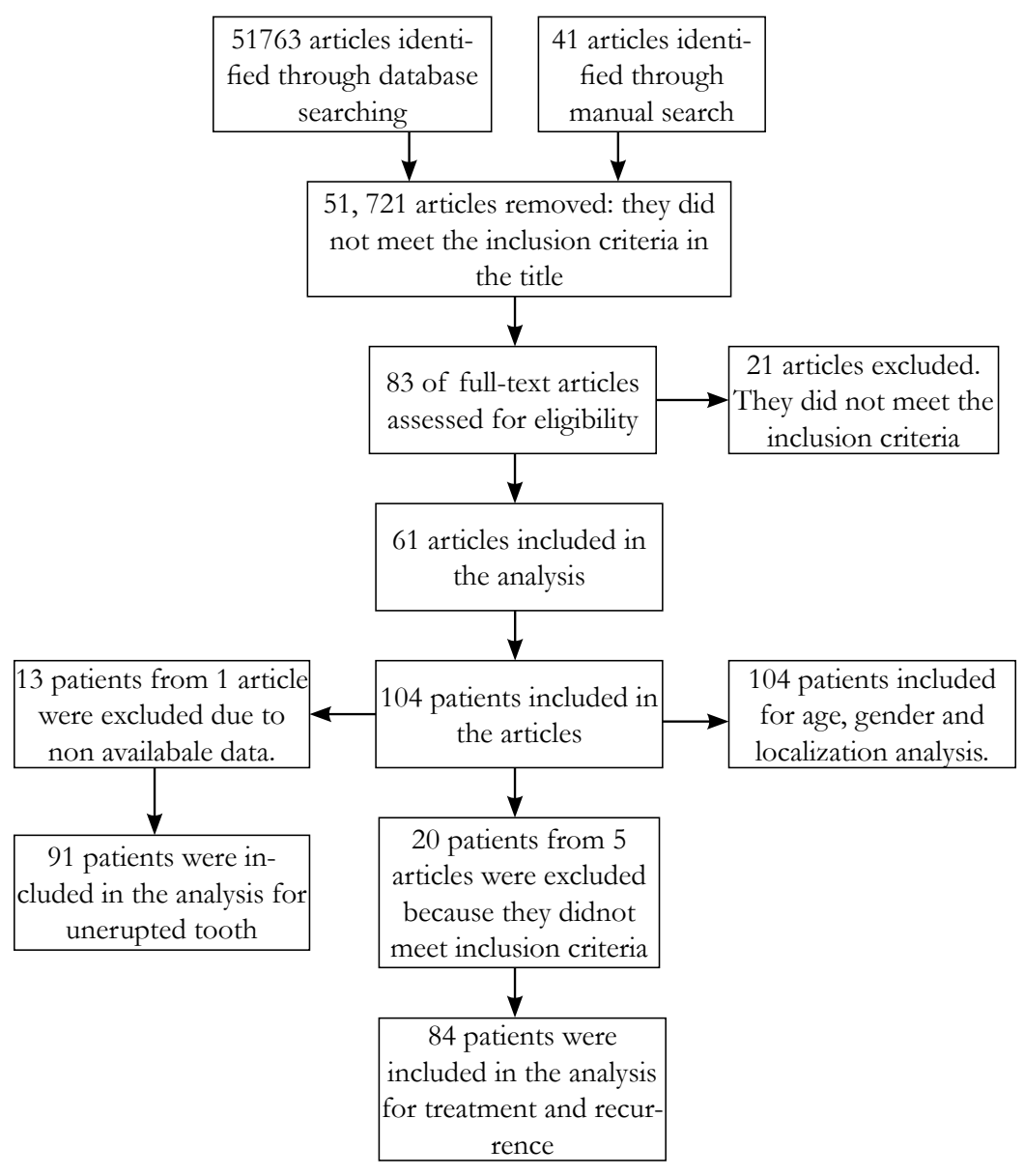


Eighty-Four patients were included in the analysis.

The age of the patients ranged from 3 to 74 years old, with a mean age of 31.11 years $(n=104)$, with a gender relation of $0.79: 1$ (46 males - 58 females).

The unerupted toot relation for the 91 patients was $28.57 \%$ (26 patients).

The most frequent localization for all patients was the mandible in $52 \%$ (54 patients), and the maxilla 48\% (50 patients).
The surgery type of the 84 patients included were as shown in the Table 2.

The recurrence of the 84 patients was $4.76 \%$ (4 patients) Table 3.

\section{Discussion}

\section{Unerupted Tooth Relation}

Cicconetti and colleagues made a literature survey which included 64 patients, from which $25 \%$ of them were associated with the

Table 1. Summary of Patients and Articles Included in the Systematic Review.

\begin{tabular}{|c|c|c|c|c|c|c|c|}
\hline Reference & Age & Gender & $\begin{array}{l}\text { Unerupted } \\
\text { tooth relation }\end{array}$ & $\begin{array}{l}\text { Localiza- } \\
\text { tion }\end{array}$ & Treatment & $\begin{array}{l}\text { Follow- } \\
\text { up }\end{array}$ & Recurrence \\
\hline Wesley RK, et al., [6] & $11 \mathrm{y}$ & $\mathrm{M}$ & Yes & Mandible & Enucleation/Curettage & $24 \mathrm{Mo}$ & No \\
\hline Heimdal A, et al., [7] & $20 \mathrm{y}$ & $\mathrm{F}$ & No & Mandible & Enucleation & $108 \mathrm{Mo}$ & Yes \\
\hline Schofield ID [8] & $11 \mathrm{Y}$ & $\mathrm{F}$ & No & Mandible & Enucleation & $\mathrm{N} / \mathrm{A}$ & $\mathrm{N} / \mathrm{A}$ \\
\hline Dahl EC, et al., [9] & $\begin{array}{l}27 \mathrm{Y} \\
80 \mathrm{Y} \\
\end{array}$ & $\begin{array}{l}\mathrm{M} \\
\mathrm{M}\end{array}$ & $\begin{array}{l}\text { No } \\
\text { Yes }\end{array}$ & $\begin{array}{c}\text { Maxilla } \\
\text { Mandible }\end{array}$ & $\begin{array}{c}\text { Enucleation } \\
\text { No treatment }\end{array}$ & $\begin{array}{l}60 \mathrm{Mo} \\
48 \mathrm{Mo} \\
\end{array}$ & $\begin{array}{c}\mathrm{No} \\
\mathrm{N} / \mathrm{A} \\
\end{array}$ \\
\hline Dunlap CL, Barker BF [10] & $\begin{array}{l}33 \mathrm{Y} \\
35 \mathrm{Y}\end{array}$ & $\begin{array}{l}\mathrm{F} \\
\mathrm{F}\end{array}$ & $\begin{array}{l}\text { No } \\
\text { No }\end{array}$ & $\begin{array}{l}\text { Maxilla } \\
\text { Maxilla }\end{array}$ & $\begin{array}{l}\text { Curettage } \\
\text { Curettage } \\
\end{array}$ & $\begin{array}{l}120 \mathrm{Mo} \\
108 \mathrm{Mo} \\
\end{array}$ & $\begin{array}{l}\text { No } \\
\text { No }\end{array}$ \\
\hline Janssen JH, Blijdorp PA [11] & $44 \mathrm{Y}$ & $\mathrm{M}$ & No & Maxilla & Enucleation & $6 \mathrm{Mo}$ & No \\
\hline $\begin{array}{c}\text { Sepheriadou- Mavropoulou } \\
\text { TH, et al., [12] }\end{array}$ & $16 \mathrm{Y}$ & $\mathrm{F}$ & No & Maxilla & Enucleation & $30 \mathrm{Mo}$ & No \\
\hline Watt-Smith SR, et al., [13] & $63 \mathrm{Y}$ & $\mathrm{F}$ & No & Mandible & Enucleation & $9 \mathrm{Mo}$ & No \\
\hline Handlers JP, et al., [14] & $\begin{array}{l}14 \mathrm{Y} \\
72 \mathrm{Y} \\
28 \mathrm{Y} \\
45 \mathrm{Y} \\
41 \mathrm{Y} \\
23 \mathrm{Y} \\
26 \mathrm{Y} \\
28 \mathrm{Y} \\
30 \mathrm{Y} \\
22 \mathrm{Y} \\
42 \mathrm{Y} \\
61 \mathrm{Y} \\
14 \mathrm{Y} \\
46 \mathrm{Y} \\
24 \mathrm{Y} \\
66 \mathrm{Y} \\
39 \mathrm{Y} \\
52 \mathrm{Y} \\
34 \mathrm{Y} \\
\end{array}$ & $\begin{array}{l}\text { F } \\
\text { M } \\
\text { M } \\
\text { F } \\
\text { F } \\
\text { F } \\
\text { F } \\
\text { F } \\
\text { F } \\
\text { F } \\
\text { F } \\
\text { F } \\
\text { F } \\
\text { F } \\
\text { M } \\
\text { F } \\
\text { F } \\
\text { F } \\
M \\
\end{array}$ & $\begin{array}{l}\mathrm{N} / \mathrm{A} \\
\mathrm{N} / \mathrm{A} \\
\mathrm{N} / \mathrm{A} \\
\mathrm{N} / \mathrm{A} \\
\mathrm{N} / \mathrm{A} \\
\mathrm{N} / \mathrm{A} \\
\mathrm{N} / \mathrm{A} \\
\mathrm{N} / \mathrm{A} \\
\mathrm{N} / \mathrm{A} \\
\mathrm{N} / \mathrm{A} \\
\mathrm{N} / \mathrm{A} \\
\mathrm{N} / \mathrm{A} \\
\mathrm{N} / \mathrm{A} \\
\mathrm{N} / \mathrm{A} \\
\mathrm{N} / \mathrm{A} \\
\mathrm{N} / \mathrm{A} \\
\mathrm{N} / \mathrm{A} \\
\mathrm{N} / \mathrm{A} \\
\mathrm{N} / \mathrm{A} \\
\end{array}$ & $\begin{array}{c}\text { Maxilla } \\
\text { Maxilla } \\
\text { Maxilla } \\
\text { Maxilla } \\
\text { Maxilla } \\
\text { Maxilla } \\
\text { Maxilla } \\
\text { Maxilla } \\
\text { Maxilla } \\
\text { Maxilla } \\
\text { Maxilla } \\
\text { Maxilla } \\
\text { Maxilla } \\
\text { Maxilla } \\
\text { Maxilla } \\
\text { Mandible } \\
\text { Maxilla } \\
\text { Mandible } \\
\text { Mandible } \\
\end{array}$ & $\begin{array}{l}\text { Enucleation } \\
\text { Enucleation } \\
\text { Enucleation } \\
\text { Enucleation } \\
\text { Enucleation } \\
\text { Enucleation } \\
\text { Enucleation } \\
\text { Enucleation } \\
\text { Enucleation } \\
\text { Enucleation } \\
\text { Enucleation } \\
\text { Enucleation } \\
\text { Enucleation } \\
\text { Enucleation } \\
\text { Enucleation } \\
\text { Enucleation } \\
\text { Enucleation } \\
\text { Enucleation } \\
\text { Enucleation } \\
\end{array}$ & $\begin{array}{l}3 \text { Mo } \\
12 \text { Mo } \\
12 \text { Mo } \\
12 \text { Mo } \\
36 \mathrm{Mo} \\
\text { N / A } \\
\text { N / A } \\
\text { N / A } \\
\text { N / A } \\
\text { N / A } \\
\text { N / A } \\
\text { N / A } \\
\text { N / A } \\
\text { N / A } \\
\text { N / A } \\
\text { N / A } \\
\text { N / A } \\
\text { N / A } \\
\text { N / A }\end{array}$ & $\begin{array}{l}\text { No } \\
\text { No } \\
\text { No } \\
\text { No } \\
\text { No } \\
\text { N / A } \\
\text { N / A } \\
\text { N / A } \\
\text { N / A } \\
\text { N / A } \\
\text { N / A } \\
\text { N / A } \\
\text { N / A } \\
\text { N / A } \\
\text { N / A } \\
\text { N / A } \\
\text { N / A } \\
\text { N / A } \\
\text { N / A } \\
\end{array}$ \\
\hline Gunhan O, et al., [15] & $55 \mathrm{Y}$ & $\mathrm{F}$ & $\mathrm{N} / \mathrm{A}$ & Maxilla & Enucleation & $60 \mathrm{Mo}$ & No \\
\hline Thomopoulos G, et al., [16] & $53 \mathrm{Y}$ & $\mathrm{M}$ & No & Maxilla & Enucleation & $6 \mathrm{Mo}^{+}$ & $\mathrm{No}+$ \\
\hline Chen CH, Huang YL [17] & $32 \mathrm{Y}$ & $\mathrm{M}$ & $\mathrm{N} / \mathrm{A}$ & Mandible & Enucleation & $36 \mathrm{Mo}$ & No \\
\hline Allen CM, et al., [18] & $\begin{array}{l}66 \mathrm{Y} \\
14 \mathrm{Y} \\
30 \mathrm{Y}\end{array}$ & $\begin{array}{l}\mathrm{F} \\
\mathrm{F} \\
\mathrm{F}\end{array}$ & $\begin{array}{c}\text { No } \\
\text { N / A } \\
\text { No }\end{array}$ & $\begin{array}{l}\text { Maxilla } \\
\text { Maxilla } \\
\text { Maxilla }\end{array}$ & $\begin{array}{l}\text { Curettage } \\
\text { Curettage } \\
\text { Curettage }\end{array}$ & $\begin{array}{l}6 \mathrm{Mo} \\
48 \mathrm{Mo} \\
14 \mathrm{Mo} \\
\end{array}$ & $\begin{array}{l}\text { No } \\
\text { No } \\
\text { Yes }\end{array}$ \\
\hline Huey MW, et al., [19] & $42 Y$ & $\mathrm{M}$ & No & Maxilla & Enucleation & $4 \mathrm{Mo}$ & No \\
\hline Mosqueda-Taylor, et al., [20] & $17 \mathrm{Y}$ & $\mathrm{F}$ & No & Mandible & Curettage & $72 \mathrm{Mo}$ & No \\
\hline Calvo N, et al., [21] & $61 \mathrm{Y}$ & M & No & Maxilla & Enucleation & $36 \mathrm{Mo}$ & No \\
\hline Daniels JSM [22] & $30 \mathrm{Y}$ & $\mathrm{F}$ & Yes & Mandible & Enucleation & $60 \mathrm{Mo}$ & No \\
\hline Covani U, et al., [23] & $26 \mathrm{Y}$ & $\mathrm{F}$ & No & Maxilla & Enucleation & $72 \mathrm{Mo}$ & No \\
\hline Cicconetti A, et al., [24] & $17 \mathrm{Y}$ & $\mathrm{F}$ & $\mathrm{Si}$ & Maxilla & Enucleation & $24 \mathrm{Mo}$ & No \\
\hline $\begin{array}{c}\text { Cercadillo-Ibarguren I, et al., } \\
{[25]}\end{array}$ & $38 \mathrm{Y}$ & $\mathrm{F}$ & $\mathrm{Si}$ & Mandible & Enucleation / Curettage & $18 \mathrm{Mo}$ & No \\
\hline
\end{tabular}




\begin{tabular}{|c|c|c|c|c|c|c|c|}
\hline Silva CO, et al., [26] & $45 \mathrm{Y}$ & $\mathrm{F}$ & No & Mandible & Enucleation & $12 \mathrm{Mo}$ & No \\
\hline Chuang GP, et al., [27] & $20 \mathrm{Y}$ & $\mathrm{F}$ & No & Mandible & Enucleation / Curettage & $8 \mathrm{Mo}$ & No \\
\hline Araki M, et al., [28] & $40 \mathrm{Y}$ & $\mathrm{M}$ & Yes & Mandible & Enucleation & $84 \mathrm{Mo}$ & No \\
\hline Daskala I, et al., [29] & $71 \mathrm{Y}$ & $\mathrm{M}$ & No & Mandible & Enucleation & $1 \mathrm{Mo}$ & No \\
\hline Kishino M, et al., [30] & $29 \mathrm{Y}$ & M & No & Maxilla & Enucleation & $14 \mathrm{Mo}$ & No \\
\hline Brazao-Silva Mt, et al., [31] & $28 \mathrm{Y}$ & M & No & Mandible & Curettage & $156 \mathrm{Mo}$ & No \\
\hline Melo AR, et al., [32] & $16 \mathrm{Y}$ & M & No & Maxilla & Enucleation & $60 \mathrm{Mo}$ & Yes \\
\hline de-Matos FR, et al., [33] & $36 \mathrm{Y}$ & $\mathrm{F}$ & No & Maxilla & Enucleation & $11 \mathrm{Mo}$ & No \\
\hline $\begin{array}{c}\text { Mosqueda-Taylor A, et al., } \\
{[34]}\end{array}$ & $\begin{array}{l}39 \mathrm{Y} \\
48 \mathrm{Y} \\
42 \mathrm{Y} \\
17 \mathrm{Y} \\
25 \mathrm{Y} \\
14 \mathrm{Y} \\
14 \mathrm{Y} \\
49 \mathrm{Y} \\
31 \mathrm{Y} \\
28 \mathrm{Y} \\
24 \mathrm{Y} \\
39 \mathrm{Y} \\
51 \mathrm{Y} \\
25 \mathrm{Y}\end{array}$ & $\begin{array}{l}\text { M } \\
M \\
F \\
F \\
F \\
M \\
M \\
M \\
F \\
M \\
M \\
F \\
F \\
F\end{array}$ & $\begin{array}{l}\text { No } \\
\text { No } \\
\text { No } \\
\text { No } \\
\text { No } \\
\text { No } \\
\text { No } \\
\text { No } \\
\text { No } \\
\text { No } \\
\text { No } \\
\text { No } \\
\text { No } \\
\text { No }\end{array}$ & $\begin{array}{c}\text { Maxilla } \\
\text { Maxilla } \\
\text { Mandible } \\
\text { Mandible } \\
\text { Maxilla } \\
\text { Mandible } \\
\text { Mandible } \\
\text { Maxilla } \\
\text { Maxilla } \\
\text { Mandible } \\
\text { Maxilla } \\
\text { Maxilla } \\
\text { Mandible } \\
\text { Maxilla }\end{array}$ & $\begin{array}{l}\text { Enucleation } \\
\text { Enucleation } \\
\text { Enucleation } \\
\text { Enucleation } \\
\text { Enucleation } \\
\text { Enucleation } \\
\text { Enucleation } \\
\text { Enucleation } \\
\text { Enucleation } \\
\text { Enucleation } \\
\text { Enucleation } \\
\text { Enucleation } \\
\text { Enucleation } \\
\text { Enucleation }\end{array}$ & $\begin{array}{c}3 \mathrm{Mo} \\
12 \mathrm{Mo} \\
48 \mathrm{Mo} \\
156 \mathrm{Mo} \\
6 \mathrm{Mo} \\
16 \mathrm{Mo} \\
24 \mathrm{Mo} \\
6 \mathrm{Mo} \\
48 \mathrm{Mo} \\
6 \mathrm{Mo} \\
\mathrm{N} / \mathrm{A} \\
\mathrm{N} / \mathrm{A} \\
\mathrm{N} / \mathrm{A} \\
\mathrm{N} / \mathrm{A}\end{array}$ & $\begin{array}{l}\text { No } \\
\text { No } \\
\text { No } \\
\text { No } \\
\text { No } \\
\text { No } \\
\text { No } \\
\text { No } \\
\text { No } \\
\text { No } \\
\text { N / A } \\
\text { N / A } \\
\text { N / A } \\
\text { N / A }\end{array}$ \\
\hline Nah KS [35] & $17 \mathrm{Y}$ & M & No & Mandible & Enucleation & $24 \mathrm{Mo}$ & No \\
\hline Kiklander S, et al., [36] & $9 \mathrm{Y}$ & $\mathrm{M}$ & Yes & Mandible & Enucleation & $12 \mathrm{Mo}$ & No \\
\hline Bologna-Molina R, et al., [37] & $14 \mathrm{Y}$ & $\mathrm{M}$ & No & Mandible & Enucleation / Curettage & $24 \mathrm{M}$ & No \\
\hline Ahmadi SK, et al., [38] & $16 \mathrm{Y}$ & $\mathrm{M}$ & No & Mandible & Enucleation & $36 \mathrm{Mo}$ & No \\
\hline Hrichi R, et al., [39] & $\begin{array}{l}17 \mathrm{Y} \\
30 \mathrm{Y} \\
18 \mathrm{Y} \\
38 \mathrm{Y} \\
12 \mathrm{Y} \\
16 \mathrm{Y} \\
11 \mathrm{Y} \\
17 \mathrm{Y}\end{array}$ & $\begin{array}{l}\mathrm{M} \\
\mathrm{M} \\
\mathrm{F} \\
\mathrm{F} \\
\mathrm{M} \\
\mathrm{M} \\
\mathrm{F} \\
\mathrm{M}\end{array}$ & $\begin{array}{l}\text { Yes } \\
\text { Yes } \\
\text { Yes } \\
\text { Yes } \\
\text { Yes } \\
\text { Yes } \\
\text { Yes } \\
\text { Yes }\end{array}$ & $\begin{array}{c}\text { Mandible } \\
\text { Mandible } \\
\text { Maxilla } \\
\text { Mandible } \\
\text { Mandible } \\
\text { Mandible } \\
\text { Maxilla } \\
\text { Mandible }\end{array}$ & $\begin{array}{c}\text { Enucleation/Curettage } \\
\text { Enucleation/Curettage } \\
\text { Enucleation / Curettage } \\
\text { Enucleation / Curettage } \\
\text { Enucleation / Curettage } \\
\text { Enucleation / Curettage } \\
\text { Enucleation / Curettage } \\
\text { Enucleation / Curettage }\end{array}$ & $\begin{array}{l}60 \mathrm{Mo} \\
60 \mathrm{Mo} \\
60 \mathrm{Mo} \\
60 \mathrm{Mo} \\
60 \mathrm{Mo} \\
60 \mathrm{Mo} \\
60 \mathrm{Mo} \\
60 \mathrm{Mo}\end{array}$ & $\begin{array}{l}\text { No } \\
\text { No } \\
\text { No } \\
\text { No } \\
\text { No } \\
\text { No } \\
\text { No } \\
\text { No }\end{array}$ \\
\hline Hara M, et al., [40] & $\begin{array}{l}24 \mathrm{Y} \\
12 \mathrm{Y}\end{array}$ & $\begin{array}{l}\mathrm{F} \\
\mathrm{F}\end{array}$ & $\begin{array}{l}\text { No } \\
\text { Yes }\end{array}$ & $\begin{array}{c}\text { Maxilla } \\
\text { Mandible }\end{array}$ & $\begin{array}{l}\text { Enucleation } \\
\text { Enucleation }\end{array}$ & $\begin{array}{l}12 \mathrm{Mo} \\
28 \mathrm{Mo}\end{array}$ & $\begin{array}{l}\text { No } \\
\text { No }\end{array}$ \\
\hline Chhabra V, Chhabra B. [41] & $16 \mathrm{Y}$ & $\mathrm{F}$ & No & Mandible & Enucleation & $12 \mathrm{Mo}$ & No \\
\hline Kimura T, et al., [42] & $74 \mathrm{Y}$ & $\mathrm{F}$ & No & Mandible & Marginal Mandibulectomy & $48 \mathrm{Mo}$ & No \\
\hline Takeota T, et al., [43] & $29 \mathrm{Y}$ & $\mathrm{M}$ & Yes & Mandible & Enucleation & $24 \mathrm{Mo}$ & $\mathrm{No}$ \\
\hline Pushpanshu J, et al., [4] & $18 \mathrm{Y}$ & $\mathrm{F}$ & No & Mandible & Curettage & $30 \mathrm{Mo}$ & No \\
\hline Iordanidis S, et al., [44] & $39 \mathrm{Y}$ & $\mathrm{F}$ & Yes & Mandible & Enucleation / Curettage & $12 \mathrm{Mo}$ & No \\
\hline Salgado H, et al., [2] & $24 \mathrm{Y}$ & $\mathrm{M}$ & No & Maxilla & Enucleation & $18 \mathrm{Mo}$ & No \\
\hline Sachdeva SK, et al., [45] & $18 \mathrm{Y}$ & $\mathrm{F}$ & Yes & Mandible & Enucleation & $12 \mathrm{Mo}$ & Yes \\
\hline Gopinathan PA, et al., [46] & $51 \mathrm{Y}$ & $\mathrm{M}$ & No & Mandible & Enucleation & $36 \mathrm{Mo}$ & No \\
\hline Batson JP, et al., [47] & $18 \mathrm{Y}$ & $\mathrm{M}$ & No & Mandible & Enucleation / Curettage & $18 \mathrm{Mo}$ & No \\
\hline Schussel JL, et al., [48] & $54 \mathrm{Y}$ & $\mathrm{F}$ & No & Maxilla & Enucleation & $6 \mathrm{Mo}$ & No \\
\hline Venugopal S, et al., [49] & $49 \mathrm{Y}$ & $\mathrm{M}$ & No & Mandible & Enucleation & $12 \mathrm{Mo}$ & No \\
\hline Chrcanovic BR, et al., [50] & $7 \mathrm{Y}$ & $\mathrm{M}$ & Yes & Mandible & Enucleation & $9 \mathrm{Mo}$ & No \\
\hline Liu X, et al., [51] & $41 \mathrm{Y}$ & $\mathrm{M}$ & No & Maxilla & Enucleation & $13 \mathrm{Mo}$ & No \\
\hline Thankappan P, et al., [52] & $10 \mathrm{Y}$ & M & Yes & Mandible & Enucleation & $12 \mathrm{Mo}$ & No \\
\hline Soolari A, Khan A [5] & $53 \mathrm{Y}$ & M & No & Mandible & Enucleation / Curettage & $12 \mathrm{Mo}+$ & $\mathrm{No}+$ \\
\hline Hedge U, Rekha M [53] & $35 \mathrm{Y}$ & M & No & Maxilla & Enucleation & $24 \mathrm{Mo}$ & No \\
\hline Salehinejad J, et al., [54] & $10 \mathrm{Y}$ & M & $\mathrm{N} / \mathrm{A}$ & Mandible & Right Hemimandibulectomy & $14 \mathrm{Mo}$ & No \\
\hline Shiraishi T, et al., [55] & $3 \mathrm{Y}$ & $\mathrm{F}$ & Yes & Mandible & $\begin{array}{l}\text { Left Segmentary Mandibulec- } \\
\text { tomy }\end{array}$ & $24 \mathrm{Mo}$ & No \\
\hline Anbiaee N, et al., [56] & $4 \mathrm{Y}$ & M & Yes & Mandible & $\begin{array}{c}\text { Right Segmental Mandibular } \\
\text { Resection }\end{array}$ & $6 \mathrm{Mo}$ & No \\
\hline Monteiro LS, et al., [57] & $74 \mathrm{Y}$ & $\mathrm{F}$ & No & Mandible & $\begin{array}{c}\text { Er:YAG }(2940 \mathrm{~mm}) / \text { enuclea- } \\
\text { tion/Curettage }\end{array}$ & $48 \mathrm{Mo}$ & No \\
\hline
\end{tabular}




\begin{tabular}{|c|c|c|c|c|c|c|c|}
\hline El-Harti K, et al., [58] & $15 \mathrm{Y}$ & $\mathrm{F}$ & Yes & Maxilla & Enucleation & 12 Mo & No \\
\hline Santoro A, et al., [59] & $12 \mathrm{Y}$ & $\mathrm{M}$ & Yes & Mandible & Enucleation & $12 \mathrm{Mo}$ & No \\
\hline Prakash U, et al., [60] & $45 \mathrm{Y}$ & $\mathrm{F}$ & No & Mandible & Enucleation/Curettage & $6 \mathrm{Mo}$ & No \\
\hline Nakamura Y, et al., [61] & $6 \mathrm{Y}$ & $\mathrm{F}$ & Yes & Maxilla & Enucleation & $96 \mathrm{Mo}$ & No \\
\hline Pippi R, et al., [62] & $16 \mathrm{Y}$ & $\mathrm{F}$ & Yes & Mandible & Enucleation/Curettage & $60 \mathrm{Mo}$ & No \\
\hline García-Cano E, et al., [64] & $15 \mathrm{Y}$ & $\mathrm{F}$ & Yes & Mandible & $\begin{array}{c}\text { Enucleation/Curettage/Liquid } \\
\text { Nitrogen }\end{array}$ & $12 \mathrm{Mo}$ & No \\
\hline
\end{tabular}

* Y: Years; M: Male; F: Female; N/A: Non Available; Mo: Months; +: Information asked through e-mail to author.

Table 2. Summary Data of the Patients Included in the Systematic Review.

\begin{tabular}{|c|c|}
\hline Total number of patients & 104 \\
\hline Averageage of patients & 31.11 years \\
\hline Gender relation (Male:Female) & $0.79: 1$ \\
\hline Unerupted tooth relation (n=91) & $26(28.57 \%)$ \\
\hline Localization (n=104): & $54(52 \%)$ \\
Mandible & $50(48 \%)$ \\
Maxilla & \\
Surgery type (n= 84): & $53(63.09 \%)$ \\
Enucleation & $8(9.52 \%)$ \\
Curettage & $17(20.23 \%)$ \\
Enucleation and Curettage & $1(4.76 \%)$ \\
Resection procedure & $1(1.19 \%)$ \\
Er:YAG 2940mm and Enucleation and Curettage & $34.17 \mathrm{Mo}$ \\
Enucleation and Curettage and liquid nitrogen & 2.84 years \\
\hline Mean follow -up time (n=84) & $4(4.76 \%)$ \\
\hline Recurrence (n=84) & \\
\hline
\end{tabular}

$*_{\mathrm{n}}=$ number of patients

Table 3. Procedures Perform in the Recurrence Cases.

\begin{tabular}{|c|c|}
\hline Recurrence $(\mathrm{n}=84)$ & $4(4.76 \%)$ \\
\hline Type of surgery (4): & \\
Enucleation & $3(66.66 \%)$ \\
Curettage & $1(33.33 \%)$ \\
\hline
\end{tabular}

$\mathbf{n}=$ number of patients

presence of an unerupted tooth [24]. However, in our systematic review, we included 91 patients, and only 26 of them correlated with an unerupted tooth $(28.57 \%)$, while the rest did not (65 patients, $71.43 \%$ ). Therefore, its presence is not necessary to make a differential radiological diagnosis.

\section{Localization}

The localization of these tumors varies in the literature. The larger the number of patients, the more the percentage changes. Handlers described 39 cases of COF, reporting 56\% occurring in the maxilla and $44 \%$ in the mandible [14]. Ramer showed an incidence ratio in mandible and maxilla of 1:1 (34:34 patients) [64]. Meanwhile, in the most recent review by Cicconetti, they reported 52 patients, founding $61.53 \%$ in the mandible and $38.47 \%$ in the maxilla [24].
Nonetheless in our systematic review, out of 104 patients, 54 $(52 \%)$ were located in the mandible, while the rest $50(48 \%)$ were located in the maxilla.

\section{Treatment}

The first authors who reported curettage as a first-line treatment were Dunlap and Barker, who presented two cases of maxillary odontogenic fibroma treated by this method with a follow-up of 9 to 10 years without evidence of recurrence [10]. Since these lesions readily separate from their bony crypt and show no evidence of bony infiltration [5], generally the treatment described in the literature has been conservative.

However, there are special cases in which resections are necessary due to the extension of the tumor that compromises the bony structures, thus not allowing a conservative treatment; such 
treatments include mandibulectomy, hemimandibulectomy, resection and reconstruction with bone grafts or free flaps [42, 54-56]. As the technology and new treatments are discovered, various methods have been implemented for treating this tumor and to lower its recurrence, such as laser treatment described by Monteiro LS [57], or the use of liquid nitrogen as described by Garcia-Cano et al., [63].

In this review $63 \%$ (53 patients) of the patients were treated only by enucleation, followed by enucleation and curettage with $20.2 \%$ (17 patients), and only curettage in $9.5 \%$ (8 patients). Only $7.14 \%$ were treated by other methods due to the tumor extension.

\section{Recurrence}

The recurrence reports vary in the literature. The most recent review by Garcia-Cano et al., of 27 cases ( 23 treated by enucleation and 4 by a resection procedure) with a mean follow up of 20.73 months, showed no recurrence whatsoever [63]. Ramer and colleagues reported only 5 cases of recurrence. However, only 39 cases out of 68 were followed up [64].

In this systematic review out of 84 patients, 4 (4.7\%) presented recurrence. From these, 3 cases were treated by enucleation and one by curettage (Table 3 ).

Heimdal and colleagues explained that the recurrence presented with their patient may have resulted due to incomplete removal [7]. On the other hand, Allen did not describe a possible cause [18]. Melo described that mistakes made in the histological diagnosis of the tumor and an inadequate surgical technique are considered to be possible causes for recurrence; they also described that a probable explanation would be the maintenance of the impacted maxillary right canine and its periodontal ligament as a tumor reactivating factor [32]. Sachdeva SK, et al, described the most recent case for recurrence, mainly due to a probable incomplete removal of the tumor [45].

\section{Conclusion}

The COF is an extremely rare benign neoplasm found only in $0.1 \%$ of all odontogenic tumors.

The recurrence rate in these tumors is very low and mainly related to an incomplete resection. Therefore, conservative surgery must be done as a first-line treatment.

\section{References}

[1]. Muñóz-Torres C, Reyes- Olave P, Álvarez-Novoa C, Venegas-Rojas B (2010) Central odontogenic fibroma, WHO type. One case report and review of the literature. Rev Esp Cir Oral Maxifolic. 32(4): 159-164.

[2]. Salgado H, Mesquita $P$ (2014) Central odontogenic fibroma of the maxilla - A case report. Rev port Estomatol Med Dent cir Maxilofac. 55(1): 49-54.

[3]. Cortés-Castillo G, Liceaga-Reyes R, Mosqueda-Taylor A (2011) Uncommon mandibular lesión of central odontogenic fibroma combined with mandibular central giant cell granuloma. Rev Odont Mex. 15(2): 126-131.

[4]. Pushpanshu K, Kaushik R, Punyani SR, Jasuja V, Raj V, et al., (2013) Concurrent central odontogenic broma (WHO Type) and traumatic bone cyst: report of a rare case. Quant Imaging Med Surg. 3(6): 341-346.

[5]. Soolari A, Khan A (2014) Central Odontogenic Fibroma of the Gingiva: A Case Report. Open Dent J. 8: 280-288.

[6]. Wesley RK, Wysocki GP, Mintz SM (1975) The central odontogenic fibroma. Clinical and morphologic studies. 40(2): 235-245.
[7]. Heimdal A, Isacsson G, Nilsson L (1980) Recurrent central odontogenic fibroma. Oral Surg. 50(2): 140-145.

[8]. Schofield ID (1981) Central odontogenic fibroma: report of a case. J Oral Surg. 39(3): 218-220.

[9]. Dahl EC, Wolfson SH, Haugen JC (1981) Central odontogenic fibroma: review of literature and report of cases. J Oral Surg. 39(2): 120-124.

[10]. Dunlap CL, Barker BF (1984) Central odontogenic fibroma of the WHO type. Oral Surg oral Med Oral pathol. 57(4): 390-395.

[11]. Janssen JH, Blijdorp PA (1985) Central Odontogenic Fibroma. A case report. J Max Fac Surg. 13(5): 236-238.

[12]. Sepheriadou-Mavropoulou TH, Patrikikou A, Sotiriadou S (1985) Central odontogenic fibroma. Int J Oral Surg. 14(6): 550-555.

[13]. Watt-Smith SR, Ell-Labban NG, Tinkler SM (1988) Central odontogenic fibroma. Int J Oral Maxillofac Surg. 17(2): 87-91.

[14]. Handlers JP, Abrams AM, Melrose RJ, Danforth R (1991) Central Odontogenic Fibroma: Clinicopathologic Features of 19 Cases and Review of the Literature. J Oral Maxillofac Surg. 49(1): 46-72.

[15]. Gunhan O, Gurbuzer B, Gardner DG, Demiriz M, Finci R (1991) A central odontogenic fibroma exhibiting pleomorphic fibroblasts and numerous calcifications. Br J Oral Maxillofac Surg. 29(1): $42-43$.

[16]. Thomopoulos G, Markopoulos AK (1992) Central odontogenic fibroma: a case report. Ann Dent. 51(2): 12-13.

[17]. Chen Ch, Huang YL (1992) Central odontogenic fibroma of the mandible a case report. Gaoxiong Yi Xue Ke Xue Za Zhi. 8(6): 327-331.

[18]. Allen CM, Hammond HL, Simson PG (1992) Central odontogenic fibroma, WHO type. A report of three cases with unusual associated giant cell reaction. Oral Surg Oral Med Oral Pathol. 73(1): 62-68.

[19]. Huey MW, Bramwell D, Hutter JW, Kratochvil FJ (1995) Central Odontogenic Fibroma Mimicking a Lesion of Endodontic Origin. J Endod. 21(12): 625-627.

[20]. Mosqueda-Taylor A, Bermúdez-Flores V, Díaz-Franco MA (1999) Combined Central Odontogenic Fibroma and Giant Cell Granuloma-Like Lesion of the Mandible: Report of a Case and Review of the Literature. J Oral Maxillofac Surg. 57(10): 1258-1262.

[21]. Calvo N, Alonso D, Prieto M, Junquera L (2002) Central Odontogenic Fibroma Granular Cell Variant: A Case Report and Review of the Literature. J Oral Maxillofac Sur. 60(10): 1192-1194.

[22]. Daniels JS (2004) Central odontogenic fibroma of mandible: A case report and review of the literature. Oral Surg Oral Med Oral Pathol Oral Radiol Endod. 98(3): 295-300.

[23]. Covani U, Crespi R, Perrini N, Barone A (2005) Central odontogenic fibroma: A case report. Med Oral Patol Oral Cir Bucal. 10(2): E154-157.

[24]. Cicconetti A, Bartoli A, Tallarico M, Maggiani F, Santaniello S (2006) Central odontogenic fibroma interesting the maxillary sinus. Minerva stomatol. A case report and literature. 55(4): 1-7.

[25]. Cercadillo-Ibarguren I, Berini-Aytés L, Marco-Molina V, Gay-Escoda C (2006) Locally aggressive central odontogenic fibroma associated to an inflammatory cyst: a clinical, histological and immunohistochemical study. J Oral Pathol Med. 35(8): 513-516.

[26]. Silva CO, Sallum AW, Gomes-do-Couto-Filho CE, Costa-Pereira AA, Costa-Hanemann JA, et al., (2007) Localized Gingival Enlargement Associated With Alveolar Process Expansion: Peripheral Ossifying Fibroma Coincident With Central Odontogenic Fibroma. J Periodontal. 78(7): 1354-1359.

[27]. Chuang GP, Tsai LL (2008) Central Odontogenic Fibroma of Mandible - A Case Report. Taiwan J Oral Maxillofac Surg. 19: 179-185.

[28]. Araki M, Nishimura S, Matsumoto N, Ohnishi M, Ohki H, et al., (2009) Central odontogenic fibroma with osteoid formation showing atypical radiographic appearance. Dentomaxillofac Radiol. 38(6): 426-430.

[29]. Daskala I, Kalyvas D, Kolokoudias M, Vlachodimitropoulos D, Alexandridis C (2009) Central odontogenic fibroma of the mandible: a case report. J Oral Sci. 51(3): 457-461.

[30]. Kishino M, Ishibashi M, Koizumi H, Sato S, Murakami S, et al., (2009) Central odontogenic fibroma of the maxilla: a case report with immunohistochemical study. Oral Med Pathol. 14(1): 29-32.

[31]. Brazao-Silva MT, Fernandez AV, Durighetto-Junior AF, Cardoso SV, Loyola AM (2010) Central odontogenic fibroma: a case report with long-term follow-up. Head Face Med. 6: 20.

[32]. Melo AR, Santos T-deS,- do-Amaral MF, Albuquerque D-de-P, Andrade ES (2011) Recurrence of central odontogenic fibroma: A rare case. General dentistry. Gen Dent. 59(2): e78-81.

[33]. de-Matos FR, de-Moraes M, Capistrano-Neto A, de-Costa-Miguel MC, Dantas-da-Silveira EJ (2011) Central odontogenic fibroma. Ann Diagno Pathol. 15(6): 481-484.

[34]. Mosqueda-Taylor A, Martínez-Mata G, Carlos-Bregni R, Agustin-Vargas P, Toral-Rizo V, et al., (2011) Central odontogenic fibroma: new findings and report of a multicentric collaborative study. Oral Surg Oral Med Oral Pathol Oral Radiol Endod. 112(3): 349-358. 
[35]. Nah KS (2011) Central odontogenic fibroma: a case report. Imaging Sci Dent. 41(2): 85-86.

[36]. Niklander S, Martínez R, Deichier J, Esguep A (2011) Bilateral mandibular odontogenic fibroma (WHO type): Report of a case with 5-year radiographic follow-up. J Dental Sci. 6(2): 123-127.

[37]. Bologna-Molina R, Pacheco-Ruiz L, Mosqueda-Taylor A, Huesca-Ramírez HG, Ponce-Lonato JA, et al., (2011) Central Odontogenic Fibroma combined with Central Giant Cell Lesion of the Mandible. Immunohistochemical profile. J Clin Exp Dent. 3(4): e348-351.

[38]. Ahmadi SK, Rahpeyma A (2012) Central Odontogenic Fibroma of the Mandible. JDMT. 1(2): 70-73.

[39]. Hrichi R, Gargallo-Albiol J, Berini-Aytés, Gay-Escoda C (2012) Central odontogenic fibroma: Retrospective study of 8 clinical cases. Med Oral Patol Oral Cir Bucal. 17(1): e50-e62.

[40]. Hara M, Matsuzaki H, Katase N, Yanagi Y, Unetsubo T, et al., (2012) Central odontogenic fibroma of the jawbone: 2 case reports describing its imaging features and an analysis of its DCE-MRI findings. Oral Surg Oral Med Oral Pathol Oral Radiol. 113(6): e51-e58.

[41]. Chhabra V, Chhabra A (2012) Central odontogenic broma of the mandible. Contemp Clin Dent. 3(2): 230-3.

[42]. Kimura T, Ohba S, Yoshimura H, Katase N, Imamura Y, et al., (2013) Epithelium-Poor Type Central Odontogenic Fibroma: An Immunohistological Study and Review of the Literature. J Hard Tissue Biology. 22(2): 273-278.

[43]. Takeoka T, Inui M, Okumura K, Nakamura S, Shimizu K, et al., (2013) A central odontogenic fibroma mimicking a dentigerous cyst associated with an impacted mandibular third molar - Immunohistological study and review of literature. J Oral Maxillofaci Surg. Medi Pathol. 25(2): 193-196.

[44]. Iordanidis S, Poulopoulos A, Epivatianos A, Zouloumis L (2014) Central odontogenic fibroma: Report of case with immunohistochemical study. Indian J Dent Res. 24(6): 753-755.

[45]. Sachdeva SK, Verma P, Verma D, Verma KG (2014) Recurrent central odontogenic fibroma: An uncommon clinical presentation. Saudi Surg J. 2(1): $22-25$.

[46]. Gopinathan PA, Kokila G, Jyothi M, Nair MS, Jacob TH, et al., (2014) Importance of Histopathology in Diagnosis of Central Odontogenic Fibroma - A Case Report. Res J Pharma, Biol Chem Sci. 5(6): 923-928.

[47]. Batson JP, Strickland F (2014) Central Odontogenic Fibroma: Case Report and Review. US Army Med Dep J. 57-60.

[48]. Schussel JL, Gallottini MHC, Braz-Silva PH (2014) Odontogenic fibroma WHO-type simulating periodontal disease: Report of a case. J Indian Soc Periodont. 18(1): 85-87.

[49]. Venugopal S, Radhakrishna S, Raj A, Sawhney A (2014) Central odontogenic fibroma. J Indian soc Periodontol. 18(2): 240-243.

[50]. Chrcanovic BR, Freire-Maia B, Gómez RS (2014) Small Central Odontogenic Fibroma Mimicking Hyperplastic Dental Follicle and Dentigerous Cyst. J Maxillofac Oral Surg. 13(3): 332-336.

[51]. Liu X, Yan S, Liu J (2014) Neurovascular involvement in central odontogenic fibroma: a potential source of confusion with invasive carcinoma. Histopathology. 66: 1035-1053.

[52]. Thankappan P, Chundru NSV, Amudala R, Yanadi P, Rahamthullah SAKU, et al., (2014) Central Odontogenic Fibroma of Simple Type. Case rep dent.

[53]. Hedge U, Rekha M (2015) Central odontogenic fibroma of maxilla - A case report. Int J Appl Dental Sci. 1(4): 05-07.

[54]. Salehinejad J, Ghazi N, Heravi F, Ghazi E (2015) Concurrent central odontogenic fibroma (WHO type) and odontoma: Report of a rare and unusual entity. J Oral Maxillofac Surg. Medi Pathology. 27(6): 888-892.

[55]. Shiraishi T, Uehara M, Fujita S, Ikeda T, Asahina I (2015) A case of central odontogenic fibroma in a pediatric patient: Mandibular reconstruction with parietal bone. J Oral Maxillofaci Surg Medic Pathol. 27(3): 361-365.

[56]. Anbiaee N, Ebrahimnejad H, Sanaei A (2015) Central odontogenic fibroma (simple type) in a four-year-old boy: atypical cone-beam computed tomographic appearance with periosteal reaction. Imaging Sci Dent. 45(2): 109115 .

[57]. Monteiro LS, Martins M, Pacheco JL, Salazar F, Magalhaes J, et al., (2015) Er:YAG Laser Assisted Treatment of Central Odontogenic Fibroma of the Mandible. Case Rep Dent. DOI: http://dx.doi.org/10.1155/2015/230297.

[58]. El-Harti K, Oujilal A, El-Wady W (2015) Central odontogenic fibroma of the maxilla. Indian J Dent. 6(4): 217-220.

[59]. Santoro A, Pannone G, Ramaglia L, Bufo P, Lo-Muzio L, et al., (2016) Central odontogenic fibroma of the mandible: A case report with diagnostic considerations. Ann med surg. 5: 14-18.

[60]. Prakash U, Shapoo Mi, Chauhan R (2016) Central Odontogenic Fibroma: A Case report. J Adv Med Dent Scie Res. 4(2): 60-62.

[61]. Nakamura Y, Masuda K, Yamaza H, Nonaka K (2016) Central Odontogenic Fibroma Associated with Eruption Disturbance of a Permanent Incisor in a Six-Year-Old Girl. Int J Dentistry Oral Sci. 03(3): 205-208.

[62]. Pippi R, Santoro M, Patini R (2016) The central odontogenic fibroma: How difficult can be making a preliminary diagnosis. J clin Exp Dent. 8(2): e223225.

[63]. García-Cano E, González-Magaña F, Malagón-Hidalgo HO, Caicedo-Perez JK , Carrillo-Ponce CS, et al., (2016) Central odontogenic fibroma: collection of cases in the world literature. Case report with use of adyuvant treatment for the reduction of recurrence with liquid nitrogen and bone regeneration guided with lyophilized bone and platelet-rich plasma. Int J Dentistry Oral Sci. S3: 002, 11-15.

[64]. Ramer M, Buonocore P, Krost B (2002) Central odontogenic fibroma - report of a case and review of the literature. Period Clin Invest. 24(1): 27-30.

Special Issue on
"Dental Abnormalities and Oral Health"
Theme Edited by:
Faiez-N. Hattab, Jordan
University of Science and Technology, Jordan.
E-Mail: f_hattab@hotmail.com

